\title{
Tangence
}

\section{Bible et art}

\section{Gérard Blais}

Numéro 35, mars 1992

Des écritures à lire

URI : https://id.erudit.org/iderudit/025702ar

DOI : https://doi.org/10.7202/025702ar

Aller au sommaire du numéro

Éditeur(s)

Tangence

ISSN

0226-9554 (imprimé)

1710-0305 (numérique)

Découvrir la revue

Citer ce document

Blais, G. (1992). Bible et art. Tangence, (35), 105-111.

https://doi.org/10.7202/025702ar d'utilisation que vous pouvez consulter en ligne.

https://apropos.erudit.org/fr/usagers/politique-dutilisation/ 


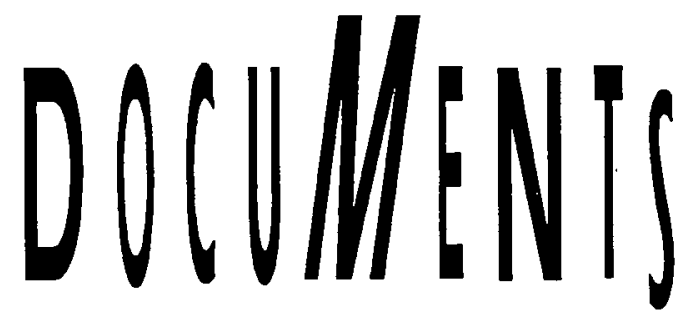

\section{Bible et art}

\section{Gérard Blais}

Depuis quelques années, j'offre à mes étudiants de niveau collégial un cours sous le titre *Bible et art. Le trajet suivi est triple: tantôt nous partons des arts pour aller vers la Bible, tantôt nous partons de la Bible pour aller vers les arts, et la session se termine par la production d'une œuvre d'art. Étant donnée la nature du cours, il me paraît plus logique de commencer par l'exégèse de quelques textes, pour vérifier aussitôt les applications dans le domaine des arts. Au fur et à mesure que nous avançons dans la session, la Bible devient alors un réservoir de créativité pour les étudiants. Les réflexions qui vont suivre portent sur cette expérience à la fois exaltante et prometteuse.

La Bible est certainement une des plus riches références culturelles de l'Occident. La plupart des chefs-d'œuvre produits en Europe ou en Amérique ont une connotation biblique. Nous sommes à même de vérifier, ces jours-ci, la pertinence de cette observation, en regardant les reportages qui nous parviennent de l'URSS. Sous le régime communiste, on a produit des édifices si peu esthétiques que le seul fond de scène un peu présentable pour la télévision étrangère demeure la somptueuse église de Basile-le-Bienheureux à Moscou ou la cathédrale Saint Paul de Saint-Petersbourg (alias Léningrad). On dirait que la pensée du -petit Père des peuples " a littéralement stérilisé la créativité alors que la Bible avait engendré une production artistique proprement géniale. De ce côté-ci de la lune, les plus beaux monuments, les plus belles créations ont été et demeurent d'inspiration religieuse et très souvent biblique. 
106

En ce qui regarde l'histoire du Québec, la Bible a servi de matrice à son imaginaire iconographique et un peu littéraire ${ }^{1}$. Il suffit de visiter la chapelle des Ursulines et de faire une tournée des églises rurales pour se rendre compte de l'impact de la Bible dans la peinture et la sculpture du début de la colonie jusqu'à nos jours. De larges segments du Québec artistique sont tributaires de la Bible. Dans bien des cas, tenant compte du fait que les traductions françaises étaient rares et que le clergé catholique n'encourageait guère la lecture de l'Ancien Testament, les artistes euxmêmes devaient se rabattre sur leur manuel d'Histoire sainte ${ }^{2}$. Cela a pourtant suffi à les lancer sur la voie de grandes ouvres.

Qu'observons-nous aujourd'hui? Nous assistons à la disparition silencieuse de la Bible comme référence culturelle de la jeune génération. Ce premier virage a été amorcé, me semble-t-il, par le Mouvement laïc de langue française, vers les années 1960, dans cette période de mutation sociale profonde au Québec, plus connue sous l'appellation de * Révolution tranquille. En déblatérant à propos du *Petit catéchisme*, on a eu tendance à reléguer la Bible aux oubliettes. Même dans la catéchèse où s'opérait pourtant une recherche vigoureuse et prometteuse sous la conduite de Jacques Laforêt, on s'est mis à préférer les chansons de Jacques Brel et de Jean Ferrat au texte biblique. Ajoutons que la pédagogie des *feuilles volantes * au détriment des manuels a accentué cette distance que nous commencions à prendre vis-à-vis du Livre. Malgré certains efforts, mais peut-être à cause des changements successifs de programme, les jeunes des années soixante-dix connaissaient moins la Bible que leurs grands-parents avec leur Petite bistoire sainte. Aujourd'hui, les élèves terminent leur secondaire avec un bagage très limité de connaissances bibliques et ce phénomène a peut-être pris de l'ampleur avec le choix des cours de morale. : La Bible? Connais pas. " Voilà une remarque étonnante entendue pour la première fois, dans mon collège, en 1991.

1 On peut penser à Félix Leclerc, Lionel Groulx, Louis Hémon, FélixAntoine Savard, etc. encore que nous soyons plus dans un univers religieux que proprement biblique.

2 Thérèse de Lisieux n'a jamais eu une Bible entre les mains. Les seuls textes qu'elle connaissait lui étaient parvenus à travers la liturgie et par sa soeur Céline. Cette dernière, plus âgée que Thérèse, s'occupait de leur père malade. Elle recopiait pour sa soeur entrée au Carmel à 15 ans des sections de la Bible que lui enseignait le Père Pichon, son directeur spirituel. 
Quand on ne connaît la Bible que par bribes, il arrive aussi qu'on s'en amuse. Petit à petit, l'esquive du sacré nous a entraînés sur la pente du comique. . C'tait just' pour rire!. Puisque tout devait être mesuré à l'aune du fun, on ne s'est pas privé de faire usage de la Bible dans une publicité qui devint vite insignifiante. -Femme, que votre règne arrive! * Fréquentez notre club Nautilus, au nom du corps... et du sain esprit!. Une génération de Datsun fut vendue sous le plagiat du Notre Père, sans oublier les * dix commandements de la CSST. Puisqu'on en était rendu là, autant envoyer un moine en bure franciscaine pour livrer une pizza baptisée * Le péché du gourmand! * Dans le judaïsme, on blêmirait à la pensée d'utiliser les textes sacrés pour une annonce de saucisses. Dans l'islam, chacun sait qu'il risque la pendaison à commenter quelques versets douteux du Coran ${ }^{3}$.

Nos grands-parents avaient probablement une meilleure connaissance générale de la Bible à la fin de leur primaire que nos étudiants à la fin de leurs études collégiales. Je crains que bientôt une bonne partie des jeunes Québécois ne connaîtront l'histoire de l'arche de Noé qu'à travers les annonces publicitaires de Snap-Seal ${ }^{4}$; ils bénéficieront d'une vague réminiscence de la Genèse en consultant la publicité du "Musée du Fjord* où l'on voit émerger du Saguenay, une soucoupe contenant le Musée avec ces paroles qui parodient la Bible: -Dieu dit: "Que le Musée du Fjord soit! Et Dieu vit que cela était bon. " Comme si les musées s'étaient donné le mot, les usagers de la CTCUQ ont eu droit pendant plusieurs mois à une imitation de la fresque de Michel-Ange dans la Sixtine, par le biais d'un poster du Père Éternel que les autobus ont baladé à travers la ville de Québec pour mousser les collections de son musée.

À vrai dire, exposer la Bible au musée ne me gêne guère. Au contraire. Mais que se passe-t-il entre les visites de musées? Estce qu'on a le droit de priver la jeune génération de ce qui lui appartient comme héritage? Si la Bible appartient au patrimoine mondial, comme on se plaît à le dire, il doit bien appartenir aussi à notre patrimoine national! En somme, il devient urgent de rapatrier une pièce importante de notre civilisation judéo-chrétienne, - de se réapproprier la Bible au titre de référence culturelle,

3 Salman Rushdie, The Satanic Verses, New York, Viking, 1988, 547 p.

$4 S N A P-S E A L$, Le système de gouttières en vinyle le plus perfectionné offert sur le marché! Cf. L'actualité. 
108

réservoir pour l'imaginaire, matrice mythologique des productions de l'esprit . 5 .

Je ne veux quand même pas être trop alarmiste, car j'observe un regain d'intérêt pour les arts bibliques. Il convient de signaler quelques belles réalisations qui ont pris racines chez nous. Tout d'abord FIDEART, le * Centre-Dieu des arts et de la culture ", mis sur pied par le père Armand Chouinard, pianiste réputé, qui avait déjà fondé ALPEC dans les années soixante-dix. FIDEART est la réponse à l'invitation lancée par Jean-Paul II à l'Université Laval en 1984: "N'acceptez jamais le divorce entre la foi et la culture." Chaque dimanche dans la chapelle historique des sœurs du Bon Pasteur, dans le vieux Québec, la *Messe des artistes * réunit des gens de talent qui aident à prier sur du beau.

Qui n'a pas entendu parler également du festival de Lanaudière? La région de Joliette regorge d'artistes de tout poil, musiciens, peintres, écrivains qui ont trouvé une expression vigoureuse à leur talent dans la foulée des Lindsay, Corbeil, Brunelle, des Max Boucher, René Pageau, Bruno Hébert, etc., tous de la communauté des Clercs de Saint-Viateur. Un simple passage dans la région de Joliette nous laisse ivres de culture comme une abeille qui a butiné follement dans une prairie gorgée de pollen. Un simple regard sur la programmation nous révèle les préoccupations du festival: du classique et du spirituel, toujours imprégné de la Bible. Peu d'espaces culturels auront attiré pour la même soirée des personnalités aussi marquantes que Don Helder Camara et l'Abbé Pierre. Il conviendrait également de mentionner L'évangile en papier de Claude Lafortune, un produit de chez nous déjà exporté à l'étranger pour faire connaître la Bible aux tout petits. Tout près de nous, à StJean-Port-Joli, il y a quelques années, un projet original a réuni plusieurs sculpteurs autour d'une création collective: la réalisation d'une crèche de Noël représentant des personnages de notre milieu. Et puis survient une exposition, un concert, un anniversaire, une manifestation culturelle un peu racée, voilà qu'on expose un ange polychrome de Louis Jobin, on redore un retable des. Baillargé et l'on interprète la Messe québécoise de Pierick Houdy. Les jeunes aussi retrouvent des éléments de la Bible. Depuis un an, ils font tourner avec une certaine ferveur Mea Culpa d'Enigma ${ }^{6}$, une

5 Anne-Marie Pelletier, “ La Bible comme mémoire culturelle ", Etudes, $n^{\circ} 3743$ (mars 1991), page 383.

6 Enigma, Curly M.C. \& David Fairstein. Distribué par Virgin Records Canada Inc., Toronto, 1990. 
musique dont les tonalités grégoriennes trouvent un écho quelque part dans l'inconscient. Enigma passera, Palestrina ne passera pas. Dans cette veine de réalisations inspirées de la Bible, le dernier-né c'est probablement Mess'aje 7 qui associe les sciences bibliques avec les moyens modernes de communication. D'ici peu, l'informatique nous réservera des surprises... ${ }^{8}$ Certes, il se fait des choses, mais il faudrait davantage. Vatican II a libéré les cultures particulières. Chaque pays doit maintenant relever le défi de l'acculturation en créant des cuvres originales?

Bible et art. Revenons-y. Au niveau collégial, nonobstant ce que je viens de mentionner, j'ai parfois l'impression de partir d'une connaissance primaire, version Snap-Seal. Alors, inutile de commencer par l'hébreu! Je verse à mes étudiants de bonnes rasades de Bible, nature. Or, ils aiment. Une page de texte lue en classe engendre cinq minutes d'un silence émouvant. La Parole sacrée s'avère supérieure à celle du professeur... Le premier contact avec la Bible se produit par le texte. Le choix du texte est à la fois important et délicat, même si les jeunes s'intéressent assez peu aux problèmes de traduction. En français, nous sommes assez bien pourvus, ce qui ne m'empêche pas de veiller au grain. Puisqu'il s'agit d'un premier contact un peu substantiel avec la Bible, je me méfie des traductions accommodantes, pour ne pas dire fantaisistes ${ }^{10}$. Le contact avec l'histoire biblique se fait

7 Mess'aje (MESSe Association Jésus Église), au service de la transmission de la foi aux adultes, est né à Lille (France) à l'initiative de Jacques Bernard. Il existe une section au Manitoba et à Québec.

8 Daniel Barric, - Futurs s, Le point, 5 octobre 1991, page 65.

9 Je me permets ici de mentionner ma modeste contribution en ce sens. Il s'agit de la mise sur pied, en septembre 1991, d'un Centre biblique au Campus Notre-Dame-de-Foy. Le but principal du Centre consiste dans une initiation à la -lecture juive - de la Bible, c'est-à-dire une appropriation de l'univers culturel de la Bible pour mieux comprendre cette Parole de Dieu comme un héritage venu du judaïsme.

10 La revue L'actualité en date du $1^{\circ}$ juin 1991 a fait état d'un essai de traduction de l'évangile en - langue québécoise : L'évangile sélon TiMarc. la controverse a éclaté dans Le devoir, sous la plume de Suzanne Martin dans son article - La Bible "traduite" ou l'abrutissement à la québécoise - (19 juin 1991, B-8). L'auteur, M. Guy Saint-Michel, a donné la réplique dans Le soleil du 15 septembre 1991. Nous reproduisons ici quelques passages de cet essai mené à l'Université Laval: .Une gang arrive avec une personne handicapée portée par quatre gars. Ils ne sont pas capables de s'approcher de Jésus à cause de la foule. Ils décident de passer par la galerie d'en arrière.' (Mc 2,1-5) Ou encore: -Quand on 
avantageusement par le cinéma. Les étudiants retiennent plus facilement l'histoire de David en visionnant l'excellent film $L e$ roi David $^{11}$ de Martin Elfand, qu'en lisant les deux livres de Samuel.

Après le texte, les cuvres. Nous en avons à profusion. Les églises du Québec, mêmè les plus reculées, regorgent d'œuvres exceptionnelles. J'envoie donc mes élèves, dans leur milieu, avec planche à dessin, caméra, magnétophone ou vidéo pour découvrir et apprécier les trésors de leur église paroissiale. Ils reviennent fascinés par tant de beauté. Le fleuron de la culture urbaine ou rurale réside dans nos églises. Elles offrent plusieurs avantages sur les musées car on trouve tout sur place: l'architecture, la peinture, la sculpture, les vitraux et la musique. Année après année, s'opère la même magie: les élèves ressentent une grande fierté face à leur patrimoine. Notons au passage, que l'émission de Radio-Canada Le jour du Seigneur joue toujours un rôle éminemment éducatif en révélant à la population, depuis plus 25 ans, les trésors de nos églises paroissiales.

La dernière étape de la démarche consiste à produire une œuvre d'inspiration biblique. J'invite mes élèves à se faire confiance et à produire quelque chose d'original. Il ne s'agit jamais de créer quelque chose de bien, mais d'excellent. Ils ont le choix du sujet et du médium; la seule condition: créer une œuvre biblique. Il arrivera qu'ils puiseront dans les quatre thèmes développés en classe: la création - la royauté (David et Salomon / musique et architecture) - Jésus - l'Apocalypse. Les sujets traités par les artistes en herbe sont très variés mais avec une prédilection pour le premier et le dernier livre de la Bible, c'est-à-dire la Création et l'Apocalypse. L'accent est souvent mis sur le tragique. L'Apocalypse hante leur imaginaire. Est-ce dû aux nombreux films de sorcellerie ou de possessions diaboliques qui s'en inspirent? Il n'est pas rare aussi que la vie des jeunes soit marquée du *signe de la bête *! La science des profondeurs pourrait en dire davantage sur le choix de ces thèmes. Je n'ai pas d'explication, seulement quelques intuitions. De toute façon, n'est-ce pas à partir du vécu

allume une lampe de poche, c'est toujours ben pas pour la cacher dans son sac de couchage. (Mc 4,21) (Claude Cossette et Guy Saint-Michel, L'bomme le plus extraordinaire que j'aie rencontré, Université Laval, Québec, 1991.)

11 King David, production anglo-italienne de Martin Elfand. Paramount Pictures, 1985. Dirigé par Bruce Beresford. 
et de la sensibilité des artistes que des œuvres géniales ont vu le jour? Comment interpréter le triptyque de Jordi Bonet au Grand théâtre de Québec, avec ses thèmes de la mort, de l'espace et de la liberté, sans les mettre en liaison avec l'histoire personnelle de l'auteur ${ }^{12}$ et l'histoire du Québec au moment de la Révolution tranquille? Tout en reflétant ses états d'âme, Jordi Bonet exprime admirablement Genèse 3 ou Apocalypse 8!

C'est ici que les arts bibliques prennent tout leur sens. Après s'être réapproprié le texte, l'artiste l'interprète. Il doit l'interpréter en le renvoyant à des questions actuelles. Rien d'alarmant si les différentes lectures ne sont pas identiques. Les croyants donneront une interprétation dans un cadre confessionnel: pour eux, ce livre dévoile le sens ultime de la vie. D'autres donneront une interprétation dans un cadre plus humaniste. Comme le disait André Beauchamp *le message culturel de la Bible repose sur la conviction que la parole est l'élément constitutif de l'être humain. Seul un être humain est capable de saisir une parole et de la traduire sur le plan éthique ou technique. Au fond, la Bible appartient à un double patrimoine: cultuel et culturel. Il est évident que le fond est religieux et amène le lecteur à un Dieu *qui n'est pas fait de mains d'homme»; mais la forme utilisée, les images et la langue, appartient à une culture spécifique. Plus que des Écritures à lire, la Bible est une Parole qui rebondit du fond de l'histoire pour éclairer notre aujourd'hui.

La réalisation artistique permet aux élèves d'exprimer quelque chose de leurs propres sentiments d'admiration, de joie, d'espérance ou d'angoisse à travers la peinture, la sculpture, la musique, le vitrail, le fusain, l'aquarelle, le théâtre, le mime et que sais-je encore. Au cours de la session, ils ont lu quelques grandes pages de la Bible; ils sont entrés en contact avec leur patrimoine; ils ont créé une première ouvre, parfois superbe, souvent modeste, rarement banale. Par-dessus tout, ils ont découvert un goût pour la Bible et les arts. Dans le coin secret d'un cégep, s'éveille peut-être l'âme d'un Baillargé, d'un Quévillon, d'un Levasseur ou d'un Dom Bellot.

12 Jordi Bonet est resté hanté par la guerre civile espagnole et le bombardement de Guernica dont Picasso fit une toile impressionnante qui lui mérita l'exil. De plus, jeune enfant, Jordi Bonet se fit amputer le bras droit qu'il s'était blessé en chutant d'un arbre. 